\title{
Epigallocatechin gallate (EGCG) esters with different chain lengths fatty acids and their antioxidant activity in food and biological systems
}

\author{
Nishani Perera, Priyatharini Ambigaipalan and Fereidoon Shahidi*
}

\author{
Department of Biochemistry, Memorial University of Newfoundland, St. John's, NL, Canada A1B 3X9 \\ *Corresponding author: Fereidoon Shahid, Department of Biochemistry, Memorial University of Newfoundland, St. John's, NL A1B 3X9, \\ Canada. Tel: 709 864-8552; E-mail: fshahidi@mun.ca \\ DOI: $10.31665 /$ JFB.2018.1131 \\ Received: January 11, 2018; Revised received \& accepted: February 14, 2018 \\ Citation: Perera, N., Ambigaipalan, P., and Shahidi, F. (2018). Epigallocatechin gallate (EGCG) esters with different chain lengths fatty \\ acids and their antioxidant activity in food and biological systems. J. Food Bioact. 1: 124-133.
}

\begin{abstract}
Ester derivatives of epigallocatechin gallate (EGCG) were prepared by acylation with different chain lengths acyl chlorides, namely acetyl chloride, C2:0; propionyl chloride, C3:0; caproyl chloride, C6:0; capryloyl chloride, C8:0; lauroyl chloride, C12:0; stearoyl chloride, C18:0; and docosahexaenoyl chloride, C22:6. The resultant products, mainly tetra-esters, were purified and their lipophilicity, radical scavenging ability and antioxidant activities in food (bulk oil, $\beta$-carotene/linoleate emulsion and ground meat) and biological systems (LDL cholesterol and DNA) evaluated. The lipophilicity of the esters increased with increasing chain length of the acyl group and also led to the enhancement of their antioxidant properties. These findings strongly suggest that the EGCG ester derivatives have great potential as lipophilic alternatives to the water-soluble EGCG.
\end{abstract}

Keywords: Epigallocatechin gallate (EGCG); Acyl chlorides; Lipohilicity; Antioxidant activity; Food systems; Biological systems.

\section{Introduction}

Tea (Camellia sinensis) is the second most highly-consumed beverage in the world after water and a rich source of dietary polyphenols. Tea is categorized into green tea (fresh devoid of oxidation/inactivation of polyphenol oxidase), oolong tea (medium oxidation) and black tea (complete oxidation) based on the degree of oxidation during processing. Catechins (flavan-3-ols) are the major polyphenols ( $\sim 30 \%$ dry weight basis) present in green tea extract, which include (-)-epicatechin (EC; $6.4 \%$ of total catechins), (-)-epicatechin-3-gallate (ECG; 13.6\%), (-)-epigallocatechin (EGC; 19\%) and (-)-epigallocatechin-3-gallate (EGCG; $59 \%$ ) (McKay and Blumberg, 2002). While green tea is rich in catechins, in black tea catechins are mostly converted to theaflavins and thearubigins by polyphenol oxidase during processing.

EGCG has been shown to exert radical scavenging, reducing and metal chelating activity in various systems such as oil-in-water emulsions and meat models (Amarowicz and Shahidi, 1995;
Sun and Ho, 2001; Xu et al., 2004; Cabrera et al., 2006; Zhong and Shahidi, 2011; Zhong et al., 2012). Wanasundara and Shahidi (1996) found that refined seal blubber oil and menhaden oil treated with dechlorophilized tea catechins had excellent oxidative stability as compared with the commonly used antioxidants, such as $\alpha$-tocopherol, butylated hydroxyanisole (BHA), butylated hydroxytolene (BHT) and tert-butylhydroquinone (TBHQ). Several epidemiological and clinical studies have shown the beneficial effects of green tea with respect to cardiovascular health and cancer (Cabrera, Artacho and Giménez, 2006; Zhong et al., 2012; Yiannakopoulou, 2014).

EGCG is a four-ring structured polyphenol and highly hydrophilic due to the presence of eight hydroxyl groups. Even though EGCG is a powerful antioxidant its hydrophilic nature limits its widespread application in various food (fats, oils, lipid based foods) and biological systems. For example, the hydrophilic nature of the molecule affects its absorption via gastrointestinal tract, metabolism and elimination from the body and also restricts its accessibility via phospholipid bilayer into mitochondrial cells, 
hence limiting its biological activity in humans. Riemersma et al. (2001) reported that EGCG and other tea catechins, after intestinal absorption, are metabolized and converted to conjugated forms as glucuronides and sulfates, predominantly in the liver, and some are methylated by catechol- $O$-methyltransferase, hence increased hydrophilicity facilitates its elimination via urine and bile. To increase the application areas and efficacy of green tea EGCG, Zhong and Shahidi $(2011 ; 2015)$ for the first-time esterified hydroxyl groups of EGCG with molecules such as stearic acid (SA; 18:0), eicosapentaenoic acid (EPA; 20:5) and docosahexaenoic acid (DHA; 22:6) in a patented process. Several promising beneficiary effects of lipophilic EGCG derivatives such as antioxidant, antiviral, anti-glycation and anti-carcinogenic effects were reported (Zhong and Shahidi, 2011; 2012; Wang et al., 2016). Myers, Fuller and Yang (2013) reported that EGCG esters could also naturally exist in green tea. In this study, a series of saturated fatty acids with varied chain length $(\mathrm{C} 2: 0, \mathrm{C} 3: 0, \mathrm{C} 6: 0, \mathrm{C} 8: 0, \mathrm{C} 12: 0, \mathrm{C} 18: 0)$ were esterified with EGCG and the influence of chain length on antioxidant capacity was evaluated. In addition, enhancement of lipophilicity with increased chain length of fatty acids as influenced by various antioxidant mechanisms in food and biological systems was explored.

\section{Materials and methods}

\subsection{Materials}

EGCG was provided by Taiyo Company (Nagoya, Japan). Acyl chlorides of different chain-length (C2:0, C3:0, C6:0, C8:0, C12:0, C18:0) were purchased from Sigma Aldrich Canada Ltd (Oakville, ON, Canada). DHA was prepared from DHA single cell oil (DHASCO) provided by DSM Corporation (Columbia, MD, USA). Other chemicals were purchased from Sigma-Aldrich Canada Ltd. (Oakville, ON, Canada). All solvents used were obtained from Fisher Scientific Ltd (Ottawa, ON, Canada). The solvents employed were of HPLC or reagent grade.

\subsection{Preparation of EGCG ester derivatives}

DHA was prepared from DHASCO by saponification followed by a urea complexation process as described by Wanasundara and Shahidi (1996). EGCG was acylated with different acyl chlorides (C2:0, C3:0, C6:0, C8:0, C12:0, C18:0 and DHA) using the method described by Zhong and Shahidi (2011). Esterification of EGCG was carried out with acyl chlorides at a mole ratio of $1: 1$ except for EGCG C2:0 and C3:0, which were prepared at a 1:2 ratio. EGCG was dissolved in ethyl acetate and acyl chloride was added dropwise to the medium containing pyridine, which removes the released $\mathrm{HCl}$ as ammonium chloride precipitates. The reaction was carried out in an oil bath under a nitrogen blanket with continuous stirring at $50{ }^{\circ} \mathrm{C}$. Upon completion of the esterification reaction, the mixture was cooled down to room temperature, filtered and washed three times with warm deionized water $\left(60^{\circ} \mathrm{C}\right)$. The ethyl acetate layer was then collected, filtered through anhydrous sodium sulfate and evaporated to obtain a dry powder of crude mixture of EGCG esters.

\subsection{Purification of EGCG derivatives}

The crude EGCG ester products were purified by flash column chromatography. EGCG esters were eluted on a silica column with a gradient mixture containing hexane/ethyl acetate/formic acid (90:10:2 to 40:60:2, v/v/v). The collected fractions were monitored by thin layer chromatography (TLC) with hexane/ethyl acetate/ formic acid (3:3:0.12, v/v/v); fractions corresponding to each band were collected and solvents removed using a rotary evaporator.

\subsection{Identification and structure elucidation of EGCG derivatives}

A reverse phase HPLC-MS, using an Agilent 1,100 HPLC unit (Agilent Technologies, Palo Alto, CA, USA) with a UV-diode array detector (UV-DAD) was used to identify the composition of crude reaction mixtures and purified compounds. A gradient elution with methanol/water mobile phase (80:20-100:0, v/v from 0 to $30 \mathrm{~min})$ was used with a SUPERLCOSILTM LC-18 column $(4.6 \times$ $250 \mathrm{~mm} \times 5 \mu \mathrm{m}$ with guard column; Sigma-Aldrich, Oakville, $\mathrm{ON}$, Canada) and fractions were detected at $280 \mathrm{~nm}$. Each fraction was further identified using a MS detector system (LC-MSD-Trap-SL, Agilent) with APCI (atmospheric pressure chemical ionization) in a positive mode.

The degree of substitution and the location of esterification of purified compounds were identified using ${ }^{1} \mathrm{H}$ and ${ }^{13} \mathrm{C}$ NMR analyses. The ${ }^{1} \mathrm{H}$ and ${ }^{13} \mathrm{C}$ spectra were recorded on a Bruker Avance $500 \mathrm{MHz}$ NMR spectrometer (Bruker Biospin Co. Billerica, MA, USA) operating at 500.13 and $125.77 \mathrm{MHz}$, respectively. Dimethyl sulfoxide (DMSO)-d6 was used to dissolve the samples and TMS was used as an internal standard. Signal processing and interpretation of the data were performed with using a Topspin 1.3 (Bruker Biospin Co.) and MestRe Nova (Mestrelab Research SL, Santiago De Compostela, Spain) software. Structure elucidation was performed by comparing the chemical shifts of EGCG derivatives with those of the EGCG parent molecule, as previously detailed (Zhong and Shahidi, 2011).

\subsection{Determination of lipophilicity}

A shake flask method using octanol-water partition coefficient $(P)$ was used to determine the lipophilicity of EGCG and its derivatives (Zhong and Shahidi, 2011). A mixture containing $100 \mathrm{~mL}$ of octanol and $100 \mathrm{~mL}$ of deionized water was shaken in a shaking water bath for 24 hours at room temperature. The content was then allowed to separate into two phases for $24 \mathrm{~h}$. Samples $(0.2 \mu \mathrm{mol})$ were dissolved in the pre-saturated octanol phase (upper phase, 5 $\mathrm{mL}$ ) and the absorbance $\left(\mathrm{A}_{0}\right)$ was read at $280 \mathrm{~nm}$. A blank without sample was prepared. Then water phase (bottom phase, $5 \mathrm{~mL}$ ) was added and the mixture was vortexed for 2 min followed by a $24 \mathrm{~h}$ standing. After separation, absorbance $\left(\mathrm{A}_{\mathrm{x}}\right)$ of the upper phase was read and the octanol-water partition coefficient $(P)$ subsequently calculated using the following equation.

$$
P=\frac{\log A_{x}}{\left(A_{0}-A_{x}\right)}
$$

\subsection{DPPH radical scavenging capacity using electron paramag- netic resonance (EPR) spectrophotometry}

The DPPH radical scavenging assay was determined following the method of Madhujith and Shahidi (2005) with slight modification. Two milliliters of $0.3 \mathrm{mM}$ solution of DPPH in ethanol were added to trolox standard solutions $(50-300 \mu \mathrm{M})$ and test compounds $(25$ $\mu \mathrm{M})$ dissolved in ethanol. Contents were mixed well and after 10 
min of reaction time, the mixture was passed through the capillary tubing to the sample cavity of a Bruker e-scan EPR spectrophotometer (Bruker E-scan, Bruker Biospin Co., Billercia, MA, USA). The spectrum was recorded after $1 \mathrm{~min}$. The operating parameters for EPR were as follows: $1.86 \mathrm{G}$ modulation amplitude, $2.621 \mathrm{~s}$ sweep time, 8 scans, $100.000 \mathrm{G}$ sweep width, $3,495 \mathrm{G}$ center field, 5.12 ms time constant, $9.795 \mathrm{GHz}$ microwave frequency, and 86 $\mathrm{kHz}$ modulation frequency. The corresponding signal intensity was used to monitor the decrease of the DPPH radical concentration in the presence of test compounds. DPPH radical scavenging activity was determined using the following equation.

$D P P H$ radical scavenging capacity (\%) =

$\{$ EPR signal intensity for the control - EPR signal intensity for the sample $\times 100$

\subsection{Antioxidant activity in bulk oil determined by a Rancimat meth- od}

A Rancimat method was used to measure antioxidant activity of EGCG derivatives in a stripped corn oil model system (Zhong and Shahidi, 2012). Samples were dissolved in ethanol $(1 \mu \mathrm{mol} / \mathrm{mL})$ and transferred $(1 \mathrm{~mL})$ into a reaction tube. The solvent was then removed under a stream of nitrogen to dryness and stripped corn oil (3 g, devoid of endogenous antioxidants) was added to each tube. A tube without any sample was used as the control. The experiment was conducted under accelerated oxidation in a Rancimat apparatus (Metrohm Model 743, Herisau, Switzerland) with an air flow rate of $20 \mathrm{~L} / \mathrm{h}$ at $100{ }^{\circ} \mathrm{C}$. Lipid oxidation in each tube was monitored by changes in electrical conductivity due to the formation of volatile oxidative compounds. The time taken to reach a sudden increase in the rate of oxidation known as induction periods (IP) was recorded. Antioxidant activity was reported as protection factor $(\mathrm{PF})$ that was calculated using following formula.

$$
P F=\frac{I P_{\text {sample }}}{I P_{\text {control }}}
$$

The IP sample and IP $_{\text {control }}$ are induction periods for the oil with test samples and induction periods for the oil without test samples, respectively.

\subsection{Antioxidant activity in a 6 -carotene/linoleate emulsion sys- tem}

Antioxidant activity (AA) of EGCG and its derivatives in $\beta$-carotene/linoleate emulsion system was examined by measuring the loss of $\beta$-carotene color in the emulsion system (Amarowicz and Shahidi, 1995). Ten milligrams of $\beta$-carotene were dissolved in $10 \mathrm{~mL}$ of chloroform; $1.2 \mathrm{~mL}$ of this solution were transferred into a round-bottom flask containing $40 \mathrm{mg}$ of linoleic acid and $400 \mathrm{mg}$ of Tween 40 . A blank devoid of $\beta$-carotene was also prepared (linoleic acid [40 mg] and Tween 40 [400 mg]). The chloroform was subsequently removed under a stream of nitrogen and oxygenated distilled water $(100 \mathrm{~mL})$ was added to the flask followed by vigorous stirring. Emulsions $(4.5 \mathrm{~mL})$ were added to test compounds ( $1 \mathrm{mM}$ in ethanol, $0.5 \mathrm{~mL})$ or ethanol as the control, vortexed and the absorbance was read at $470 \mathrm{~nm}\left(\mathrm{~A}_{0}\right.$ - absorbance at time zero). A mixture of blank was prepared for each sample. The tubes were then placed in a water bath with gentle shaking at $50{ }^{\circ} \mathrm{C}$ and absorbance was measured at $15 \mathrm{~min}$ intervals over a 105 min period. Antioxidant activity of test compounds in $\beta$ carotene/ linoleic acid emulsion was calculated using the following formula, in which $\mathrm{A}_{0}$ and $\mathrm{A}_{\mathrm{t}}$ are corrected absorbance values for test compounds at zero time and after incubation, respectively.

$$
A A \%=\left[1-\frac{A_{0}-A_{t}}{A_{0}^{\circ}-A_{t}^{\circ}}\right] \times 100
$$

\subsection{Antioxidant activity in ground meat as a muscle food system}

Antioxidant activity of EGCG and its derivatives in a muscle food system was evaluated using a pork model system as described by Shahidi and Alexander (1998). Forty grams of fresh ground pork were mixed with $10 \mathrm{~g}$ of distilled water in a Mason jar. Test compounds dissolved in ethanol were added to meat at a level of 80 $\mu \mathrm{mol} / \mathrm{kg}$, thoroughly mixed and cooked in a thermostated water bath at $80^{\circ} \mathrm{C}$ for 40 min with intermittent stirring. BHA was used as a positive control and a control without any antioxidant was also prepared. The cooked meat was then cooled to ambient temperature, homogenized with a Polytron PT 3000 (Brinkmann Instruments, Rexdale, ON, Canada) homogenizer, transferred into plastic bags and stored for 14 days at $4{ }^{\circ} \mathrm{C}$. The meat samples were removed on days $0,3,5,7$, and 14 and the formation of TBARS (thiobarbituric acid reactive substances) was measured (Shahidi and Hong, 1991). A standard curve was prepared with TMP (1,1,3,3-tetramethoxypropane). Samples and standards were mixed with TBA and heated in a boiling water bath for $45 \mathrm{~min}$. The absorbance was then recorded at $532 \mathrm{~nm}$ after cooling to room temperature. TBARS values were reported as mg malondialdehyde equivalents $/ \mathrm{kg}$ of meat. Antioxidant activity (AA) was calculated as percentage inhibition of TBARS formation by test samples.

\subsection{Inhibition of copper-induced LDL-cholesterol peroxidation}

Pure EGCG and its derivatives were evaluated for their inhibition against copper-induced LDL-cholesterol peroxidation (Lebeau et al., 2000). Samples were dissolved in ethanol $(10 \mu \mathrm{M}, 10 \mu \mathrm{L})$ and the solvent was evaporated to dryness under a stream of nitrogen. To each Eppendorf tube, PBS (10 mM, 0.15M NaCl, pH 7.4, 0.1 $\mathrm{mL}$ ) was added and vortexed. Human LDL was dialyzed overnight in PBS at $4{ }^{\circ} \mathrm{C}$ under nitrogen prior to the experiment. Dialyzed and diluted LDL solution $(0.125 \mathrm{mg} / \mathrm{m}, 0.8 \mathrm{~mL})$ was added to each tube, mixed well and incubated at $37{ }^{\circ} \mathrm{C}$ for $15 \mathrm{~min}$. The reaction was then initiated by the addition of $\mathrm{CuSO}_{4}(20 \mu \mathrm{M}, 0.1 \mathrm{~mL}, 37$ ${ }^{\circ} \mathrm{C}$ ). A blank was prepared using a sample devoid of $\mathrm{LDL}_{\text {or }} \mathrm{CuSO}_{4}$ for each test compound. The formation of conjugated dienes was measured after incubation at $37^{\circ} \mathrm{C}$ for $22 \mathrm{~h}$ using a spectrophotometer at $234 \mathrm{~nm}$. The inhibition of conjugated diene formation was calculated as follows.

$$
\% \text { Inhibition }=\frac{100 \times\left(A b s_{\text {control }}-A b s_{\text {sample }}\right)}{A b s_{\text {control }}-A b s \text { native } L D L}
$$

where the control had $\mathrm{LDL}$ and $\mathrm{CuSO}_{4}$ but devoid of antioxidants while native LDL contained only LDL.

\subsection{Inhibition against DNA scission induced by peroxyl and hy- droxyl radicals}

The inhibitory activity of EGCG and its derivatives against DNA scission was determined (Hiramoto et al., 1996). For hydroxyl 


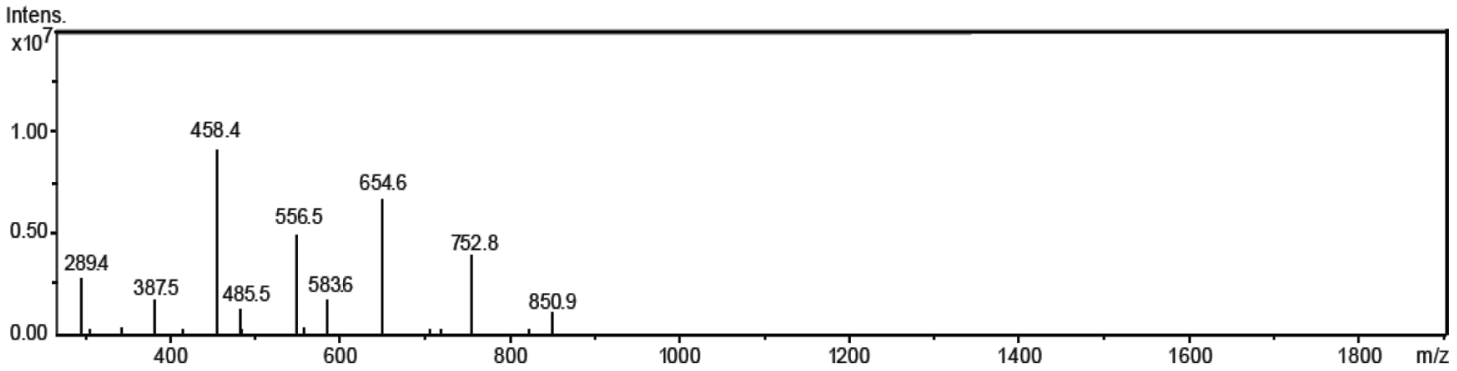

Figure 1. Mass spectrum of EGCG tetracaproylate.

radical-induced DNA oxidation, test compounds were dissolved in ethanol $(2 \mu \mathrm{L})$ and the solvent was evaporated using a stream of nitrogen. To the Eppendorf tube, deionized water $(10 \mu \mathrm{M}, 2$ $\mu \mathrm{L}$ ) was added in order to dissolve the contents by vortexing for a minute. Then PBS $(0.1 \mathrm{M}$, pH 7.4, $2 \mu \mathrm{L})$, supercoiled pBR322 DNA $(10 \mu \mathrm{g} / \mathrm{ml}, 2 \mu \mathrm{L}), \mathrm{H}_{2} \mathrm{O}_{2}(0.2 \mathrm{mM}, 2 \mu \mathrm{L})$ and $\mathrm{FeSO}_{4}(0.1$ $\mathrm{mM}, 2 \mu \mathrm{L}$ ) were added to the tube and incubated at $37{ }^{\circ} \mathrm{C}$ for 1 h. For peroxyl radical-induced DNA oxidation, test compounds (1 $\mu \mathrm{M}, 2 \mu \mathrm{L})$ were mixed with AAPH [2, 2'-azobis (2-aminopropane) dihydrochloride, $7 \mathrm{mM}, 4 \mu \mathrm{L}]$, PBS $(0.1 \mathrm{M}, 2 \mu \mathrm{L})$ and DNA (10 $\mu \mathrm{g} / \mathrm{mL}, 2 \mu \mathrm{L}$ ). A blank (DNA only) and a control (DNA+ free radicals) were used in both assays. Loading dye $(0.25 \%$ bromophenol blue $/ 0.25 \%$ xylene cyanol $/ 50 \%$ glycerol; $1 \mu \mathrm{L}$ ) was added after the incubation and the entire mixture was loaded onto an agarose gel $[0.7 \%(\mathrm{w} / \mathrm{v})$ agarose]. Agarose gel was prepared in Tris-acetic acid-EDTA (ethylenediaminetetraacetic acid) buffer (TAE, $\mathrm{pH}$ 8.5) and stained with SYBR safe. Gel electrophoresis was run in TAE buffer using a horizontal submarine gel electrophoresis apparatus (Owl Separation Systems Inc., Portsmonth, NH, USA) at $80 \mathrm{~V}$ for $90 \mathrm{~min}$. The bands were observed under UV light and a GelDoc apparatus (Cell Biosciences, Santa Clara, CA, USA) equipped with a Sony digital camera photographed the images and analyzed using Alpha Ease stand-alone software (Alpha Innotech Co., San Leandro, CA, USA). The intensity of the bands was used as an indicator of the concentration of the native (supercoiled) and nicked DNA fractions and retention percentage was calculated as follows.

$$
\% \text { Retention }=100 \times \frac{\text { native DNA sample }}{\text { native DNA blank }}
$$

The DNA sample and DNA blank in the above formula are normalized concentrations of native supercoiled DNA in total DNA for sample groups and blank, respectively.

\subsection{Statistical analysis}

Statistical analysis was performed for all test samples. All determinations were replicated three times and mean values and standard deviations reported. Analysis of variance (one-way ANOVA) was performed and the mean separations were performed by Tukey's HSD test $(\mathrm{P}<0.05)$ using SPSS 16.0 for Windows.

\section{Results and discussion}

\subsection{Purification and Identification of EGCG derivatives}

The lipophilic esters of EGCG were prepared via reaction with various acyl chlorides with crude products' yields (mainly tetra es- ters) of $67.3,66.4,74.3,65.4,56.9,45.4$ and $28.7 \%$ for $\mathrm{C} 2, \mathrm{C} 3, \mathrm{C} 6$, $\mathrm{C} 8, \mathrm{C} 12, \mathrm{C} 18$ and $\mathrm{C} 22$ esters, respectively. The yield of long-chain PUFA ester was lower compared to short chain FA due to their bent or non-linear structure, which induces a steric hindrance during the reaction, hence reduces the degree of acylation. Depending on the electrophilic nature, the reaction conditions and the type of catalysts employed the electrophilic acyl group may react with different number of hydroxyl groups located at different positions (Zhong and Shahidi, 2011). In this study, esters of EGCG with high degree of substitution (DS) were formed during acylation under the experimental conditions employed and the composition of the reaction mixture varied depending on the ratio of EGCG and fatty acid used. Lipophilicity of the EGCG derivatives increased with increased DS while accessibility of lipolytic enzymes could decrease due to steric hindrance (Akoh and Swanson, 1990). Thus, increased lipophilicity was associated with higher membrane permeability and metabolic stability of the EGCG esters. HPLC-MS analysis showed that acylation of EGCG with fatty acids predominantly produces tetra esters (DS 4) in all crude products (C6, C8, $\mathrm{C} 12, \mathrm{C} 18$ and $\mathrm{C} 22$ esters) except for $\mathrm{C} 2$ and $\mathrm{C} 3$ esters, but at various ratios. This study confirms that the chain length of the fatty acids influences their ability to incorporate into the EGCG molecule.

In this study, flash column chromatography was used to purify the EGCG esters. Isolated and purified tetra esters of each EGCGfatty acid were examined for their lipophilicity and antioxidant capacity. HPLC-MS/MS was used to identify and confirm the individual esters. A representative mass spectrum of C6-tetraester is shown in Figure. 1. For the tetra ester of C6, the molecular ion peak detected showed $\mathrm{m} / \mathrm{z}$ at 850.91 , representing $[\mathrm{M}]^{+}$ion of the EGCG-tetracaproylate, which might result from loss of a hydrogen atom from the $[\mathrm{M}+\mathrm{H}]^{+}$or its migration to the fragments. The peaks at $\mathrm{m} / \mathrm{z} 752.7,654.6,556.5$ and 458.3 showed one or multiple mass loss of 98.1 from the molecular ion, representing the ions of [M$98.1^{+},\left[\mathrm{M}-2 \times 98.1^{+}\right],\left[\mathrm{M}-3 \times 98.1^{+}\right]$and $\left[\mathrm{M}-4 \times 98.1^{+}\right]$, respectively. The peaks located at $\mathrm{m} / \mathrm{z} 583.7,485.6$ and 289.7 were due to the loss of gallic acid (mass 169) moiety corresponding to [M-2x98.1-169+ ${ }^{+}$, [M-3x98.1-169 $\left.{ }^{+}\right]$and $\left[\mathrm{M}-4 \times 98.1-169^{+}\right]$, respectively. The identity of ester was confirmed to be EGCG tetracaproylate based on these molecular ions and fragments in the mass spectrum. Similarly, all the other tetra esters were identified and confirmed for each compound (C2, C3, C8, C12, C18 and C22 esters; results not shown). A similar observation was reported by Zhong and Shahidi (2011) for EGCG esterified with stearic acid, eicosapentaenoic acid (EPA) and DHA.

\subsection{Structure elucidation of EGCG derivatives}

Since EGCG has 8 hydroxyl groups, the number of possible acylation sites is 8 . The location of fatty acid acylation with EGCG molecule was identified using ${ }^{1} \mathrm{H}$ and ${ }^{13} \mathrm{C}$ NMR, where the chemical 
Table 1. ${ }^{1} \mathrm{H}$ and ${ }^{13} \mathrm{C}$ chemical shift ( $\left.\delta \mathrm{ppm}\right)$ of epigallocatechin gallate (EGCG) and EGCG-3', 5', 3", 5"-0-tetracaproylate

\begin{tabular}{|c|c|c|c|c|}
\hline \multirow[b]{2}{*}{$\mathrm{C} / \mathrm{H}$ position } & \multicolumn{2}{|c|}{ EGCG } & \multicolumn{2}{|c|}{ EGCG-C6 } \\
\hline & ${ }^{13} \mathrm{C}$ & ${ }^{1} \mathrm{H}$ & ${ }^{13} \mathrm{C}$ & ${ }^{1} \mathrm{H}$ \\
\hline 2 & 77.66 & 4.96 & 77.59 & 4.97 \\
\hline 3 & 69.14 & 5.36 & 69.18 & 5.39 \\
\hline \multirow[t]{2}{*}{4} & 26.86 & 2.65 & 26.81 & 2.67 \\
\hline & & 2.93 & & 2.96 \\
\hline 5 & 156.8 & & 156.77 & \\
\hline 6 & 95.46 & 5.83 & 95.45 & 5.83 \\
\hline 7 & 158.62 & & 158.64 & \\
\hline 8 & 96.68 & 5.93 & 96.71 & 5.94 \\
\hline 9 & 157.61 & & 157.63 & \\
\hline 10 & 98.5 & & 98.47 & \\
\hline $1^{\prime}$ & 129.71 & & 129.61 & \\
\hline $2^{\prime}$ & 106.61 & 6.39 & 106.65 & 6.48 \\
\hline $3^{\prime}$ & 146.52 & & 150.72 & \\
\hline $4^{\prime}$ & 133.47 & & 131.77 & \\
\hline $5^{\prime}$ & 146.52 & & 150.72 & \\
\hline $6^{\prime}$ & 106.61 & 6.39 & 106.61 & 6.48 \\
\hline $1^{\prime \prime}$ & 120.42 & & 120.44 & \\
\hline $2^{\prime \prime}$ & 109.81 & 6.82 & 109.41 & 6.86 \\
\hline $3^{\prime \prime}$ & 146.76 & & 151.26 & \\
\hline $4^{\prime \prime}$ & 139.64 & & 137.14 & \\
\hline $5^{\prime \prime}$ & 146.76 & & 151.26 & \\
\hline $6^{\prime \prime}$ & 109.78 & 6.77 & 106.54 & 6.86 \\
\hline $\mathrm{COO}$ & 166.38 & & 166.36 & \\
\hline
\end{tabular}

shifts of the derivatives were compared with those of their parent EGCG molecule. The chemical shifts detected for EGCG in both ${ }^{1} \mathrm{H}$ and ${ }^{13} \mathrm{C}$ NMR were in accordance with the values reported in the literature (Valcic et al., 1999; Zhong and Shahidi, 2011). A downfield shift was observed for all proton signals of EGCG with the protons in the derivatives (Table 1). Juhel et al. (2000) reported that acetylation of catechin could displace all the proton signals towards downfield. Chemical shift displacement (6.39 to $6.48 \mathrm{ppm}$, Table 1) towards downfield in position $\mathrm{H}-2^{\prime}$ and $\mathrm{H}-6^{\prime}$ that indicates acylation of 'B' ring, while downfield shift in position H-2" (6.82 to 6.86 ppm) and H-6" (6.80 to $6.86 \mathrm{ppm}$ ) reflects acetylation of ' $\mathrm{D}$ ' ring. Zhong and Shahidi (2011) observed the same esterification position for EGCG with various fatty acids such as stearic acid, EPA and DHA. Similar positions of esterification of EGCG molecule were detected in this study. A minor change $(0.01 \mathrm{ppm})$ in $\mathrm{H}-6$ and $\mathrm{H}-8$ chemical shift indicates that there was no acylation occurring in 'A' ring. The tentative identification of acylation cites were further confirmed by ${ }^{13} \mathrm{C}$ NMR. According to Yoshimoto et al. (1980) findings, acylation of hydroxyl groups results in a downfield shift for $\mathrm{O}$-acylated carbon and an up field shift for the adjacent carbon. ${ }^{13} \mathrm{C}$ NMR results showed a downfield shift for the carbons $3^{\prime}, 5^{\prime}$ of ring B (146 to $150 \mathrm{ppm}$ ) and 3", 5" of ring D (146 to $151 \mathrm{ppm}$ ), which confirms the sites of acylation. In addition, an up field shift in C-4'

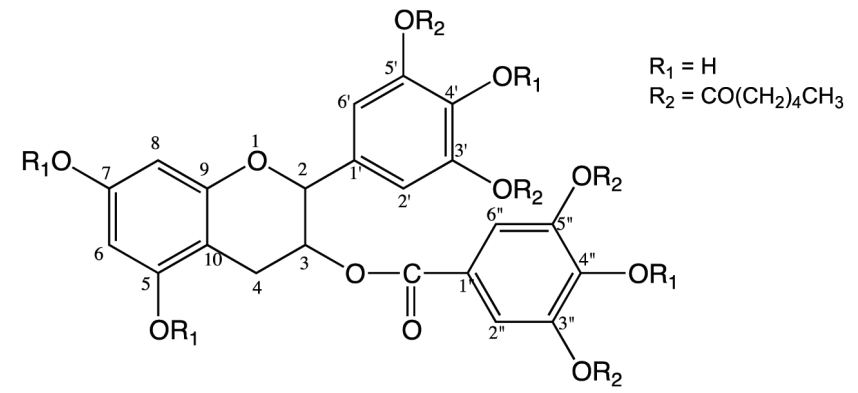

Figure 2. Chemical structures of EGCG-3',5',3",5"-O- tetracaproylate.

(133 to $131 \mathrm{ppm}$ ) and C-4" (139 to $137 \mathrm{ppm}$ ) reflects the presence of free hydroxyl groups in C-4', C-4" as well as the acylation of C-3', C-5' and C-3", C-5", respectively. Absence of up field chemical shift in other neighboring carbons could be due to the steric hindrance imposed by the fatty acid chains (Zhong and Shahidi, 2011). Detection of alkyl protons and carbon provided evidence for incorporation of fatty acids into the EGCG molecule. Based on the combined ${ }^{1} \mathrm{H}$ and ${ }^{13} \mathrm{C}$ NMR, the compound was identified as EGCG-3',5',3",5"-0- tetracaproylate, as shown in Figure 2.

\subsection{Lipophilicity}

Hydrophilicity/lipophilicity balance of bioactive molecules plays a major role in digestion and bioavailability as well as application in the food. EGCG is a highly hydrophilic molecule due to its hydroxyl groups, hence esterification could enhance its lipophilicity. Several studies have shown that acylation contributes to the increased lipophilicity of phenolic compounds such as tyrosol, hydroxytyrosol and resveratrol (Zhong and Shahidi, 2011; Oh and Shahidi, 2017; Zhou et al., 2017). The EGCG derivatives were evaluated for their lipophilicity using octanol-water partition method. Higher octanol-water partition coefficient $(P)$ values indicate higher lipophilicity of the test compounds. Lipophilicity of EGCG increased by acylation with fatty acids, which was evident from the $p$ values of esters that was greater than that of the parent EGCG molecule (Table 2). EGCG-DHA had the highest $P$ value, due to the presence of the long chain DHA moieties, while EGCG$\mathrm{C} 2$ had lowest value among the tested esters. This implies that lipophilicity increased with the increase of chain length, due to the presence of alkyl chain in fatty acids.

\subsection{Radical scavenging capacity}

The antioxidant activity of the EGCG and its esters was evaluated as their scavenging capacity against a stable, hydrophobic DPPH radical. All ester derivatives of EGCG, except EGCG-C2, displayed a higher scavenging activity against DPPH radical than the parent EGCG molecule (Figure 3 ) and the scavenging capacity increased with chain length elongation. Differences in scavenging capacity among esters were marginal but more significant $(\mathrm{P}$ $<0.05$ ) for EGCG-DHA. The DPPH radical scavenging capacity of EGCG was significantly $(\mathrm{p}<0.05)$ improved by incorporation of fatty acid chains. The observed effect was due to the increased lipophilicity by alkyl groups of fatty acids, which favored the accessibility/affinity of the derivatives to the hydrophobic DPPH radical than the parent EGCG molecule. Zhong and Shahidi (2011) reported an increase in DPPH radical scavenging activity of newly formed esters of EGCG with stearic acid, EPA and DHA. How- 
Table 2. Lipophilicity and protection factor (PF) of epigallocatechin gallate (EGCG) and esters

\begin{tabular}{llll}
\hline$\#$ & Compound* & Lipophilicity (Octanol-water partition coefficient, $P$ ) & Protection factor** $^{*}$ \\
\hline 1 & EGCG & $0.38 \pm 0.06^{\mathrm{d}}$ & $1.51 \pm 0.30^{\mathrm{c}}$ \\
2 & EGCG-C2 & $0.43 \pm 0.04^{\mathrm{a}}$ & $1.12 \pm 0.45^{\mathrm{e}}$ \\
3 & EGCG-C3 & $0.45 \pm 0.06^{\mathrm{a}}$ & $1.08 \pm 0.68^{\mathrm{e}}$ \\
4 & EGCG-C6 & $0.55 \pm 0.38^{\mathrm{a}}$ & $1.85 \pm 0.05^{\mathrm{b}}$ \\
5 & EGCG-C8 & $0.70 \pm 0.38^{\mathrm{b}}$ & $2.26 \pm 0.75^{\mathrm{a}}$ \\
6 & EGCG-C12 & $0.77 \pm 0.38^{\mathrm{b}}$ & $2.38 \pm 0.40^{\mathrm{a}}$ \\
7 & EGCG-C18 & $1.07 \pm 0.38^{\mathrm{c}}$ & $2.23 \pm 0.35^{\mathrm{a}}$ \\
\hline 8 & EGCG-DHA & $1.38 \pm 0.30^{\mathrm{c}}$ & $1.94 \pm 0.25^{\mathrm{b}}$ \\
\hline
\end{tabular}

Values (mean \pm SD of three replicates) with different letters were significantly different at $P<0.05 . *[(2)$ EGCG-C2, EGCG tetraacetate; (3) EGCG-C3, EGCG tetrapropionate; (4) EGCG-C6, EGCG tetracaproylate; (5) EGCG-C8, EGCG tetracapryloylate; (6) EGCG-C12, EGCG tetralauroylate; (7) EGCG-C18, EGCG tetrastearoylate; and (8) EGCG-DHA, EGCG tetradecosahexaenoate]. ${ }^{* *}$ In stripped corn oil at $0.5 \mu \mathrm{mol} / \mathrm{g}$ of oil.

ever, since DPPH radical is a stable artificial radical, the antioxidant activity estimated from DPPH scavenging may not necessarily reflect the real situation in food and biological systems, where radicals are generally unstable with very short life time. Thus, the antioxidant efficacy was evaluated in various food systems.

\subsection{Antioxidant activity in bulk oil}

A Rancimat method using stripped corn (absence of endogenous antioxidants) oil was employed for the detection of antioxidant activity in bulk oil system. This system generated the same effect as deep fat frying, hence the volatile compounds so produced, especially organic acids that could be detected by Rancimat. A parameter called protection factor (PF) was used to express the antioxidant capacity, which is calculated based on induction period (IP, time taken to reach a sudden increase in the rate of oxidation). The longer the IP value, the greater oxidative stability of the oil and hence high antioxidant capacity. The calculated $\mathrm{PF}$ values are shown in Table 3. EGCG showed an increase in antioxidant activity in the oil $(0.5-1 \mu \mathrm{mol} / \mathrm{g}$ of oil) than its derivatives EGCG-C2 and EGCG-C3. Meanwhile, all other derivatives of EGCG showed a significantly $(\mathrm{p}<0.05)$ greater antioxidant efficiency as reflected

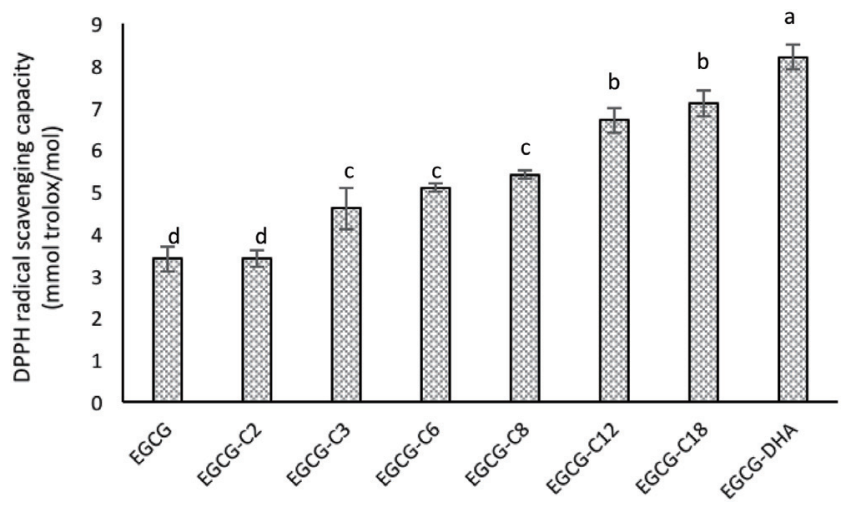

Figure 3. DPPH radical scavenging activities of epigallocatechin gallate (EGCG) and its esters. [(2) EGCG-C2, EGCG tetraacetate; (3) EGCG-C3, EGCG tetrapropionate; (4) EGCG-C6, EGCG tetracaproylate; (5) EGCG-C8, EGCG tetracapryloylate; (6) EGCG-C12, EGCG tetralauroylate; (7) EGCGC18, EGCG tetrastearoylate; and (8) EGCG-DHA, EGCG tetradecosahexaenoate]. by their PF (PF > 1) values (Table 3). The possible mechanism of EGCG involves the ability to be oriented in the air-oil interface due to their hydrophilic nature, which renders the solubility in the oil (Frankel et al., 1994). Porter (1993) reported that polar, hydrophilic antioxidants are more effective in bulk oil systems than nonpolar, hydrophobic antioxidants according to the polar paradox theory. However, in this study EGCG-C12 was most effective compared to other compounds in inhibiting oxidation while EGCG-C2 and EGCG-C3 were least effective, which reflects the influence of fatty acid chain length on their antioxidant capability in bulk oil systems. The antioxidant efficiency was increased with chain length increase and then it collapsed down with further increase in chain length. However, recent studies suggest that nonpolar antioxidants are not always more active than their polar homologues, nor polar antioxidants are more active than their nonpolar homologues (Laguerrea et al., 2011; Shahidi and Zhong, 2011), which is contradictory to the polar paradox. It was demonstrated that the relationship between antioxidant activity and hydrophobicity is not as linear as postulated by the polar paradox. For a homologous series, antioxidant activity increases with increasing of the alkyl chain length until a break point is reached, beyond which any lengthening leads to an activity collapse. This nonlinear behaviour is termed as cut-off effect and is observed in various systems whether they are living (cells) or not (lipid dispersions) (Jacobsen et al., 2008; Laguerrea et al., 2010; 2011). The common structural trait may be the lipid-water interface, and the common property may be the cut-off effect when arrived at a critical chain length. The data shows that under certain conditions and with some compounds, the effectiveness order goes in one way or the other with higher lipophilicity exerting a greater antioxidant efficiency than EGCG. With respect to compounds EGCG-C2 and EGCG-C3 with slightly less antioxidant activity in bulk oil system, this has not previously been observed and needs further studies to clarify the situation.

\subsection{Antioxidant activity in oil-in-water emulsion}

A $\beta$-carotene/linoleic acid emulsion system was used to study the antioxidant efficacy of EGCG and their derivatives in an oil/water emulsion. The loss of yellow colour of $\beta$-carotene during the coupled oxidation of linoleic acid and $\beta$-carotene was effectively prevented by EGCG and EGCG esters. The inhibition rate of $\beta$-carotene bleaching by EGCG and its derivatives varied between 13.27 and $54.49 \%$ over 105 minutes of test period (Table 2). Figure 4 shows the change in absorbance at $470 \mathrm{~nm}$ over time for the 
Table 3. Inhibitory effect of epigallocatechin gallate (EGCG) and its derivatives against $\beta$-carotene bleaching and TBARS formation in cooked ground meat

\begin{tabular}{llll}
\hline \multirow{2}{*}{ Compound* } & & Inhibition (\%) \\
\cline { 3 - 4 } & EGCG & $\beta$-carotene bleaching & TBARS formation in cooked pork \\
\hline 1 & EGCG-C2 & $20.53 \pm 0.50^{\mathrm{d}}$ & $64.45 \pm 1.65^{\mathrm{d}}$ \\
\hline 3 & EGCG-C3 & $22.24 \pm 0.45^{\mathrm{d}}$ & $66.77 \pm 0.78^{\mathrm{d}}$ \\
\hline 4 & EGCG-C6 & $40.63 \pm 0.21^{\mathrm{c}}$ & $65.43 \pm 0.65^{\mathrm{d}}$ \\
5 & EGCG-C8 & $48.25 \pm 0.34^{\mathrm{b}}$ & $88.78 \pm 0.45^{\mathrm{a}}$ \\
6 & EGCG-C12 & $54.49 \pm 0.75^{\mathrm{a}}$ & $89.65 \pm 1.23^{\mathrm{a}}$ \\
7 & EGCG-C18 & $47.26 \pm 0.24^{\mathrm{b}}$ & $90.75 \pm 0.43^{\mathrm{a}}$ \\
8 & EGCG-DHA & $46.66 \pm 0.22^{\mathrm{b}}$ & $87.45 \pm 2.45^{\mathrm{b}}$ \\
\hline
\end{tabular}

Values (mean \pm SD of three replicates) with different letters were significantly different at $\mathrm{P}<0.05 .{ }^{1}$ Inhibition (\%) calculated at the end of the incubation period (105 min). ${ }^{2}$ Inhibition (\%) calculated at the end of storage period (day 14). *[(2) EGCG-C2, EGCG tetraacetate; (3) EGCG-C3, EGCG tetrapropionate; (4) EGCG-C6, EGCG tetracaproylate; (5) EGCG-C8, EGCG tetracapryloylate; (6) EGCG-C12, EGCG tetralauroylate; (7) EGCG-C18, EGCG tetrastearoylate; and (8) EGCG-DHA, EGCG tetradecosahexaenoate].

control and test compounds, where all the test compounds exhibited higher absorbance than that of the control devoid of any antioxidant compound. All EGCG derivatives tested showed significantly higher inhibition than their parent EGCG compound due to the increased lipophilicity, among which EGCG-C12 had the highest percentage of inhibition $(\sim 55 \%)$. A similar observation was reported by Zhong and Shahidi (2012), where EGCG esterified with stearic acid showed the highest antioxidant activity followed by EGCG-DHA and EGCG-EPA. In this study, the antioxidant activity of test compounds was increased with increasing chain length up to C12 and then increase of chain length reversed the effect (Table 2). These findings further support studies carried out by others (Laguerrea et al., 2011; Shahidi and Zhong, 2011; Bayrasy et al., 2013), that is contradictory the "polar paradox" theory, which states that nonpolar, hydrophobic antioxidants are more effective than polar ones in emulsions, showing an opposite direction from that in bulk oil.

\subsection{Antioxidant activity in ground meat as a muscle model sys- tem}

EGCG and its derivatives were evaluated for their antioxidant ca-

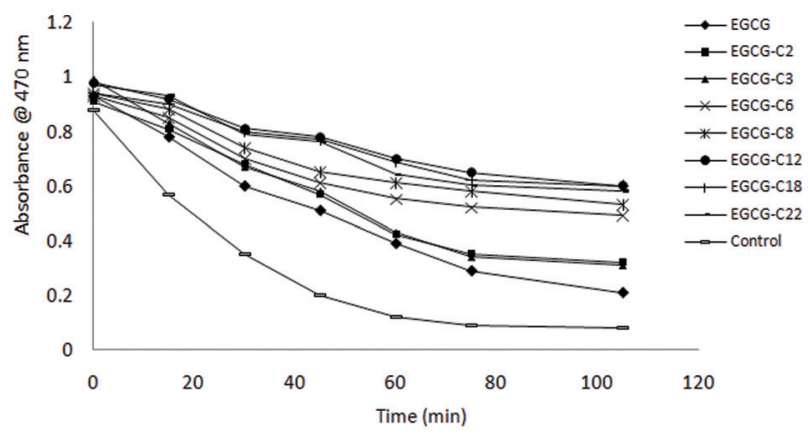

Figure 4. $\beta$-carotene bleaching as affected by epigallocatechin gallate (EGCG) and its derivatives. [(2) EGCG-C2, EGCG tetraacetate; (3) EGCG-C3, EGCG tetrapropionate; (4) EGCG-C6, EGCG tetracaproylate; (5) EGCG-C8, EGCG tetracapryloylate; (6) EGCG-C12, EGCG tetralauroylate; (7) EGCGC18, EGCG tetrastearoylate; and (8) EGCG-DHA, EGCG tetradecosahexaenoate]. pacity in cooked ground pork system, which provides an excellent model for assessing antioxidant activity in thermally processed foods. In a cooked ground pork model system, EGCG showed a higher antioxidant activity than other catechins and typical food antioxidants BHT and $\alpha$-tocopherol (Shahidi and Alexander, 1998; Zhong and Shahidi, 2012). In this assay, antioxidant capacity was measured in terms of the formation of TBARS, which reflect secondary oxidative compounds (Table 4). During storage at $4{ }^{\circ} \mathrm{C}$, TBARS were detected in all meat samples with time due to the oxidation of meat lipids. The EGCG derivatives had significantly greater antioxidant efficacy than their parent EGCG molecule. This trend is the same as the effect observed in the $\beta$-carotene bleaching assay that correlates well with the lipophilicity of EGCG and its derivatives. This results in accordance with the findings reported by Zhong and Shahidi (2012) for EGCGstearic acid, EGCG-EPA and EGC-DHA. It is evident that lipophilicity of antioxidants had a positive influence on antioxidant capacity in cooked ground pork model system. No linear relationship existed between antioxidant activity and chain length. However, the antioxidant activity of test compounds increased with increasing chain length up to EGCG-C12 and then decreased with further increase of chain length. All derivatives possessed antioxidant activity comparable to that of BHA. The results suggest that lipophilic EGCG derivatives could be used in cooked muscle foods as antioxidants.

\subsection{Inhibition of copper-induced LDL-cholesterol peroxidation}

Tea catechins, especially EGCG, have been shown to inhibit oxidation of LDL-cholesterol that could potentially lead to atherosclerosis and heart disease (Yamanaka et al. 1997; Ishikawa et al. 1997; Nakagawa et al. 1999; Trevisanato and Kim 2000; Gomikawa and Ishikawa 2002; Sajilata et al. 2008; Zhong and Shahidi, 2012). In this study, the formation of conjugated dienes during copper ion induced LDL peroxidation was measured to determine the antioxidant potential of EGCG derivatives in a biological model system. The inhibitory activity against LDL cholesterol peroxidation by EGCG and its derivatives is shown in Figure 5. All EGCG derivatives showed a significantly $(\mathrm{p}<$ 0.05 ) higher inhibition than their parent EGCG molecule. Furthermore, increased inhibition with increasing of chain length up 
Table 4. DNA retention (\%) in hydroxyl and peroxyl radical-induced oxidative scission

\begin{tabular}{llll}
\hline \multirow{2}{*}{$\#$} & \multirow{2}{*}{ Compound* $^{*}$} & \multicolumn{2}{c}{ DNA retention (\%) } \\
\cline { 3 - 4 } & & Hydroxyl radical & Peroxyl radical \\
\hline & Control & $27.87 \pm 0.45^{\mathrm{e}}$ & $23.27 \pm 0.75^{\mathrm{f}}$ \\
1 & EGCG & $43.27 \pm 1.75^{\mathrm{c}}$ & $81.45 \pm 1.65^{\mathrm{a}}$ \\
2 & EGCG-C2 & $29.53 \pm 0.50^{\mathrm{e}}$ & $56.77 \pm 0.78^{\mathrm{e}}$ \\
3 & EGCG-C3 & $30.28 \pm 0.95^{\mathrm{e}}$ & $55.43 \pm 0.65^{\mathrm{e}}$ \\
4 & EGCG-C6 & $34.43 \pm 0.21^{\mathrm{d}}$ & $68.78 \pm 0.45^{\mathrm{c}}$ \\
5 & EGCG-C8 & $48.27 \pm 0.34^{\mathrm{b}}$ & $69.65 \pm 1.23^{\mathrm{c}}$ \\
6 & EGCG-C12 & $55.49 \pm 0.85^{\mathrm{a}}$ & $70.75 \pm 0.43^{\mathrm{c}}$ \\
7 & EGCG-C18 & $40.26 \pm 1.24^{\mathrm{c}}$ & $78.45 \pm 2.45^{\mathrm{b}}$ \\
8 & EGCG-DHA & $36.65 \pm 0.22^{\mathrm{d}}$ & $64.22 \pm 1.38^{\mathrm{d}}$ \\
\hline
\end{tabular}

Values (mean \pm SD of three replicates) in the same column with different letters were significantly different at $\mathrm{P}<0.05$. *[(2) EGCG-C2, EGCG tetraacetate; (3) EGCGC3, EGCG tetrapropionate; (4) EGCG-C6, EGCG tetracaproylate; (5) EGCG-C8, EGCG tetracapryloylate; (6) EGCG-C12, EGCG tetralauroylate; (7) EGCG-C18, EGCG tetrastearoylate; and (8) EGCG-DHA, EGCG tetradecosahexaenoate].

to EGCG-C12 was observed followed by a decrease with further chain length elongation. The improved inhibitory activity of the tested derivatives against LDL oxidation may be due to their greater lipophilicity. The mechanism of inhibition could possibly be via chelation of prooxidant copper ions and scavenging of free radicals that is generated during LDL peroxidation. In addition, Satue-Gracia et al. (1997) suggested that catechins and other polyphenols could also bind to apo-lipoprotein B that induces the access of antioxidant to the lipids and prevents approaching of oxidation catalysts.

\subsection{Inhibition against DNA scission induced by peroxyl and hy- droxyl radicals}

Reactive oxygen species (ROS) such as hydroxyl and peroxyl radicals cause oxidative stress in cells which could consequently damage DNA and cause mutagenesis and carcinogenesis. Davies (1995) reported that the DNA damage could be a strand scission, sister chromatid exchange, DNA-DNA and DNA-protein cross-

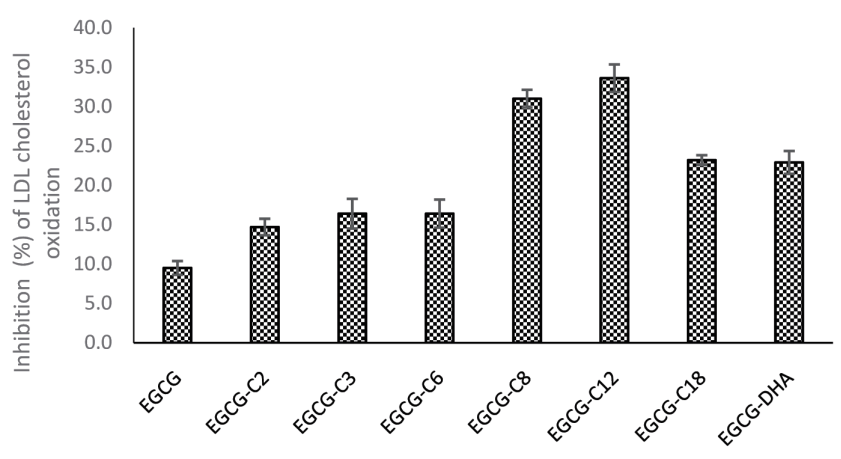

Figure 5. Inhibition of LDL choleterol peroxidation by EGC and its derivatives. [(2) EGCG-C2, EGCG tetraacetate; (3) EGCG-C3, EGCG tetrapropionate; (4) EGCG-C6, EGCG tetracaproylate; (5) EGCG-C8, EGCG tetracapryloylate; (6) EGCG-C12, EGCG tetralauroylate; (7) EGCG-C18, EGCG tetrastearoylate; and (8) EGCG-DHA, EGCG tetradecosahexaenoate].

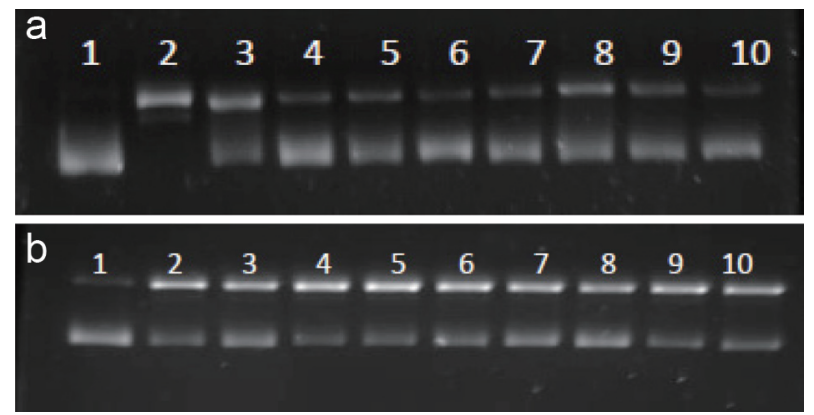

Figure 6. Inhibition of hydroxyl (A) and peroxyl (B) radicals induceed DNA scissionby EGC and its derivatives. Lanes 1-Blank (DNA only); 2-Control; 3-EGCG; 4-EGCG-C2; 5-EGCG-C3; 6-EGCG-C6; 7-EGCG-C8; 8-EGCGC12; 9-EGCG-C18; 10-EGCG-DHA. [(2) EGCG-C2, EGCG tetraacetate; (3) EGCG-C3, EGCG tetrapropionate; (4) EGCG-C6, EGCG tetracaproylate; (5) EGCG-C8, EGCG tetracapryloylate; (6) EGCG-C12, EGCG tetralauroylate; (7) EGCG-C18, EGCG tetrastearoylate; and (8) EGCG-DHA, EGCG tetradecosahexaenoate].

links, and base modification. Hydroxyl radical is the major source of ROS causing mitochondrial DNA damage (Perron et al. 2008). The inhibitory effect of EGCG and its derivatives on hydroxyl and peroxyl radicals induced DNA strand scission are shown in Figure 6 and Table 4. Lane 2 (Figure 6) represents the oxidized DNA, which is the conversion of supercoiled plasmid DNA into nicked open circular forms, hence restricting the movement. Hydroxyl radicals generated by Fenton reaction cause DNA strand scission, which was inhibited by EGCG and its derivatives, possibly via ferrous ion chelation and radical scavenging. EGCG-C8 and EGCG-C12 were more effective than EGCG against hydroxyl radical, while EGCG showed the highest inhibitory activity against peroxyl radical (Table 4). A similar observation was reported by Zhong and Shahidi (2012) for EGCG and its stearic acid, EPA and DHA esters. Previous studies have shown the inhibitory effect of EGCG against DNA scission and reported that the effect depended on the concentration of EGCG (Hiramoto et al. 1996; Ohashi et al. 2002). EGCG as a prooxidant might cause mutagenic effects on normal cells, however this might also be beneficial in terms of its cytotoxic and apoptotic effects on tumor cells (Chen et al.1998; Hadi et al. 2000). The oxidative damage of DNA varied depending on the type of the catalysts/pro-oxidants involved (e.g. free radical species, presence of metal ions), leading to different and distinct antioxidant mechanisms and activity of the test compounds in inhibiting DNA scission.

\section{Conclusion}

Lipophilization of the water-soluble epigallocatechin gallate (EGCG) via esterification with different fatty acids produced tetra esters as dominant products with enhanced lipophilicity under the experimental conditions employed. The EGCG derivatives exhibited excellent antioxidant activity in various in vitro assays, including 1,1-diphenyl-2-picrylhydrazyl (DPPH) radical scavenging activity, and showed a protective effect in various food model systems such as bulk oil, beta-carotene emulsion and ground meat. In addition, EGCG and its derivatives were effective antioxidants in various biological systems such as LDL cholesterol oxidation and DNA strand scission. Lipophilicity and chain length of fatty acid played major role in their antioxidant capacities. A synergistic effect was also noted when two bioactive compounds, EGCG and 
DHA, were joined together via covalent bonding.

\section{Acknowledgments}

We are grateful to the Natural Science and Engineering Research Council (NSERC) of Canada for financial support in the form of a Discovery grant to FS.

\section{References}

Akoh, C.C., and Swanson, B.G. (1990). Absorbability and weight gain by mice fed methyl glucoside fatty acid polyesters: potential fat substitutes. J. Nutr. Biochem. 2: 652-655. doi:10.1016/0955-2863(91)90063-B.

Amarowicz, R., and Shahidi, F. (1995). Antioxidant activity of green tea catechins in a $\beta$-carotene-linoleate model system. J. Food Lipids 2: 47-56. doi:10.1111/j.1745-4522.1995.tb00029.x.

Bayrasy, C., Chabi, B., Laguerre, M., Lecomte, J., Jublanc, E., Villeneuve, P., Wrutniak-Cabello, C., and Cabello, G. (2013). Boosting Antioxidants by Lipophilization: A Strategy to increase cell uptake and target mitochondria. Pharm. Res. 30: 1979-1989. doi:10.1007/s11095-0131041-4.

Cabrera, C., Artacho, R., and Giménez, R. (2006). Beneficial effects of green tea - A review. J. Am. Coll. Nutr. 25: 79-99. doi:10.1080/0731 5724.2006.10719518.

Chen, Z.P., Schell, J.B., Ho, C.T., and Chen, K.Y. (1998). Green tea epigallocatechin gallate show a pronounced growth inhibitory effect on cancerous cells but not in their normal counterparts. Cancer Lett. 129: 173-179. doi:10.1016/\$0304-3835(98)00108-6.

Davies, K.J.A. (1995). Oxidative stress: The paradox of aerobic life. Free radical and oxidative stress: Environments, drugs and food additives In: Rice-Evans, C.Halliwell, B., and Lunt, G.G. (Ed.). Portland Press Ltd., London, UK, pp. 1-31.

Frankel, E.N., Huang, S.W., Kanner, J., and German, J.B. (1994). Interfacial phenomena in the evaluation of antioxidants: bulk oils vs emulsions. J. Agric. Food Chem. 42: 1054-1059. doi:10.1021/jf00041a001.

Hadi, S.M., Asad, S.F., Singh, S., and Ahmad, A. (2000). Putative mechanism for anticancer and apoptosis inducing properties of plantderived polyphenolic compounds. IUBMB Life 50: 161-171. doi:10.1080/152165400300001471.

Hiramoto, K., Ojima, N., Slo, K., and Kukugawa, K. (1996). Effect of plant phenolics on the formation of the spi-adduct of hydroxyl radical and the DNA strand breaking by hydroxyl radical. Bio. Pharma. Bulletin. 19: 558-563. doi:10.1248/bpb.19.558.

Juhel, C., Armand, M., Pafumi, Y., Rosier, C., Vandermander, J., and Larson, D. (2000). Green tea extract (AR25 ${ }^{\circledR}$ ) inhibits lipolysis of triglycerides in gastric and duodenal medium in vitro. J. Nutr. Biochem. 11: 45-51. doi:10.1016/S0955-2863(99)00070-4.

Jacobsen, C., Let, M.B., Nielsen, N.S., and Meyer, A.S. (2008). Antioxidant strategies for preventing oxidative flavour deterioration of foods enriched with n-3 polyunsaturated lipids: A comparative evaluation. Trends Food Sci. Technol. 19: 76-93. doi:10.1016/j.tifs.2007.08.001.

Laguerre, M., Giraldo, L.J.L., Lecomte, J., and Figueroa-Espinoza, M.C. (2010). Relationship between hydrophobicity and antioxidant ability of "phenolipids" in emulsion: A parabolic effect of the chain length of rosmarinate esters. J. Agric. Food Chem. 58: 2869-2876. doi:10.1021/jf904119v.

Laguerre, M., Wrutniak-Cabellob, C., Chabib, B., López Giraldoa, L.J., Lecomtea, J., Villeneuvea, P., and Cabellob, G. (2011). Does hydrophobicity always enhance antioxidant drugs? A cut-off effect of the chain length of functionalized chlorogenate esters on ROS-overexpressing fibroblasts. J. Pharm. Pharmacol. 63: 531-540. doi:10.1111/j.20427158.2010.01216.x.

Lebeau, J., Furman, C., Bernier, J.L., Duriez, P., Teissier, E., and Cotelle, N. (2000). Antioxidant properties of di-tertbutylhydroxylated flavonoids. Free Radical Bio Med 29: 900-912. doi:10.1016/S08915849(00)00390-7.

Madhujith, T., and Shahidi, F. (2005). Antioxidant potential of pea beans (Phaseolus vulgaris L.). J. Food Sci. 70: S85-S90. doi:10.1111/j.1365-2621.2005.tb09071.x.

McKay, D.L., and Blumberg, J.B. (2002). The role of tea in human health: an update. J. Am. Coll. Nutr. 21: 1-13. doi:10.1080/07315724.2002. 10719187.

Myers, R.A., Fuller, E., and Yang, W. (2013). Identification of native catechin fatty acid esters in green tea (camellia sinensis). J. Agric Food Chem. 61: 11484-11493. doi:10.1021/jf403620f.

Oh, W.Y., and Shahidi, F. (2017). Lipophilization of resveratrol and effects on antioxidant activities. J. Agric. Food Chem. 65: 8617-8625. doi:10.1021/acs.jafc.7b03129.

Ohashi, Y., Yoshinaga, K., Yoshioka, H., and Yoshioka, H. (2002). Kinetic analysis of the effect of (-)-epigallocatechin gallate on the DNA scission induced by Fe(II). Biosci. Biotechnol. Biochem. 66: 770-776. doi:10.1271/bbb.66.770

Perron, N.R., Hodges, J.N., Jenkins, M., and Brumaghim, J.L. (2008). Predicting how polyphenol antioxidants prevent DNA damage by binding to iron. Inorg. Chem. 47: 6153-6161. doi:10.1021/ic7022727.

Porter, W.L. (1993). Paradoxical behavior of antioxidants in food and biological systems. Antioxidants: Chemical, Physiological, Nutritional and Toxicological Aspects In: Williams, G.M. (Ed.). Princeton Scientific, Princeton, NJ, pp. 93-122.

Riemersma, R.A., Rice-Evans, C.A., Tyrrell, R.M., Clifford, M.N., and Lean, M.E.J. (2001). Tea flavonoids and cardiovascular health. Q. J. Med. 94: 277-282. doi:10.1093/qjmed/94.5.277.

Satue-Gracia, M.T., Heinonen, M., and Frankel, E.N. (1997). Anthocyanins as antioxidants on human low-density lipoprotein and lecithin-liposome systems. J. Agric. Food Chem. 45: 3362-3367. doi:10.1021/ jf970234a.

Shahidi, F., and Alexander, D.M. (1998). Green tea catechins as inhibitors of oxidation of meat lipids. J. Food Lipid. 5: 125-133. doi:10.1111/j.1745-4522.1998.tb00112.x.

Shahidi, F., and Hong, C. (1991). Evaluation of malonaldehyde as a marker of oxidative rancidity in meat products. J. Food Biochem. 15: 97-105. doi:10.1111/j.1745-4514.1991.tb00147.x.

Shahidi, F., and Zhong, Y. (2011). Revisiting the polar paradox theory: A critical overview. J. Agric. Food Chem. 59: 3499-3504. doi:10.1021/ jf104750m.

Shahidi, F., and Zhong, Y. (2015). U.S. Patent No. 9,018,248. Washington, DC: U.S. Patent and Trademark Office.

Sun, T., and Ho, C.T. (2001). Antiradical efficiency of tea components. J. Food Lipid. 8: 231-238. doi:10.1111/j.1745-4522.2001.tb00198.x.

Valcic, S., Muders, A., Jacobsen, N.E., Liebler, D.C., and Timmermann, B.N. (1999). Antioxidant chemistry of green tea catechins. Identification of products of the reaction of (-)-epigallocatechin gallate with peroxyl radicals. Chem. Res. Toxicol. 12: 382-386. doi:10.1021/tx990003t.

Wanasundara, U.N., and Shahidi, F. (1996). Stabilization of seal blubber and menhaden oils with green tea catechins. J. Am. Oil Chem. Soc. 73: 1183-1190. doi:10.1007/BF02523382.

Wang, M., Zhang, X., Zhong, Y.J., Perera, N., and Shahidi, F. (2016). Antiglycation activity of lipophilized epigallocatechin gallate (EGCG) derivatives. Food Chem. 190: 1022-1026. doi:10.1016/j.foodchem.2015.06.033.

Xu, J.Z., Yeung, S.Y.V., Chang, Q., Huang, Y., and Chen, Z.Y. (2004). Comparison of antioxidant activity and bioavailability of tea epicatechins with their epimers. Br. J. Nutr. 91: 873-881. doi:10.1079/BJN20041132.

Yiannakopoulou, E.C. (2014). Effect of green tea catechins on breast carcinogenesis: a systematic review of in-vitro and in-vivo experimental studies. Eur. J. Cancer Prevent. 23: 84-89. doi:10.1097/ CEJ.0b013e328364f23e.

Yoshimoto, K., Itatani, Y., and Tsuda, Y. (1980). 13C-Nuclear magnetic resonance spectra of 0 -acylglucoses: additivity of shift parameters and its application to structure elucidations. Chern. Pharrn. Bull. 28: 20652074. doi:10.1248/cpb.28.2065.

Zhong, Y., and Shahidi, F. (2011). Lipophilized epigallocatechin gallate (EGCG) derivatives as novel antioxidants. J. Agric. Food Chem. 59: 6526-6533. doi:10.1021/jf201050j.

Zhong, Y., Ma, C.M., and Shahidi, F. (2012). Antioxidant and antiviral activities of lipophilic epigallocatechin gallate (EGCG) derivatives. J. Funct. Food 4: 87-93. doi:10.1016/j.jff.2011.08.003.

Zhong, Y., and Shahidi, F. (2012). Lyophilized epigallocatechin gallate 
(EGCG) derivatives and their antioxidant potential in food and biological systems. Food Chem. 131: 22-30. doi:10.1016/j.foodchem.2011.07.089.
Zhou, D.Y., Sun, Y.X., and Shahidi, F. (2017). Preparation and antioxidant activity of tyrosol and hydroxytyrosol esters. J. Funct. Foods $37: 66-73$. doi:10.1016/j.jff.2017.06.042. 